\title{
EPITAXIAL INTERGROWTH OF ISOSTRUCTURAL PHASES IN Bi-2212 SINGLE CRYSTALS
}

\author{
G.-J. BABONAS \\ Semiconductor Physics Institute, 2600 Vilnius, Lithuania \\ L. Leonyuk and V. Maltsey \\ Moscow State University, 119899 Moscow, Russia
}

\begin{abstract}
The isostructural phases in Bi-2212-type HTSC were studied by structural and optical methods. The epitaxial intergrowth was shown to be a feature typical of single crystals composed of several isostructural phases. The physical properties of the multi-phase samples were interpreted on the basis of reference mono-phase samples. A presence of the intergrowth interface was assumed to be responsible for particular features in multi-phase samples.
\end{abstract}

PACS numbers: 74.62.Bf, 74.72.Hs

A synthesis of mono-phase ceramic samples and a growth of single crystals of superconducting compounds $\mathrm{Bi}_{2} \mathrm{Sr}_{2} \mathrm{CaCu}_{2} \mathrm{O}_{8}$ with a structural type 2212 present the task which has not been completely solved up to now. Making use of a special technology [1], it is possible to obtain the 2212-type samples with no trace of co-crystallizing 2201 or other phase of different structural type. However, recently an intergrowth of the basic 2212-type orthorhombic structures was observed. The co-crystallizing phases were characterized by similar lattice parameters but different symmetries [2] or they presented the isostructural 2212-type phases of slightly different compositions [3].

In the present work the formation of isostructural 2212-type phases in single crystals of Bi-based compounds is discussed. The data obtained by X-ray diffraction, electron probe microanalysis (EPMA) and optical studies have shown that the epitaxial intergrowth of isostructural phases is a typical feature of 2212-type single crystals. The complete structure and composition data on more than 30 Bi-2212-type single crystals studied are presented in $[3,4]$.

The X-ray diffraction and spectral analyses have shown [3] that in the $\mathrm{Bi}-\mathrm{Sr}-\mathrm{Ca}-\mathrm{Cu}-\mathrm{O}$ system several 2212-type isotructural phases can be formed which differ by composition and the values of lattice constant $c$. In addition to structural studies, the optical features in the Raman spectra and in the dielectric function can be used as an indication for the presence of particular phases in the multi-phase 


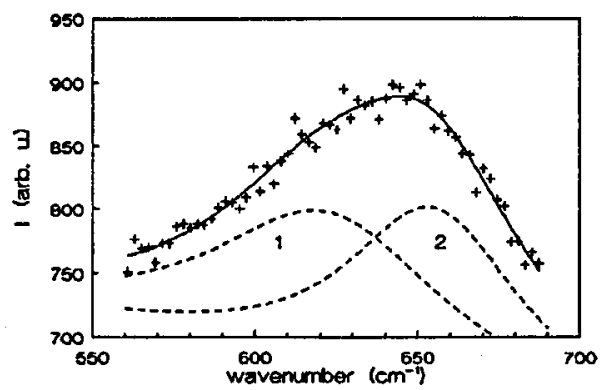

Fig. 1. Raman spectrum for multiphase 2212-type crystal (No. 1302) contributed by two modes approximated by Lorentzian lines at $622 \mathrm{~cm}^{-1}$ (1) and $655 \mathrm{~cm}^{-1}$ (2).
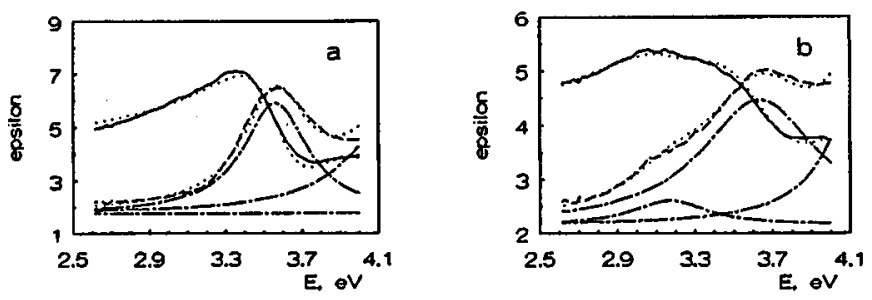

Fig. 2. Real (solid curve) and imaginary (dashed curve) parts of the dielectric function in single phase 2212-type crystals with lattice constants $a=5.421 \AA, b=27.072 \AA$, $c=30.873 \AA\left(\mathrm{Bi}_{2.00} \mathrm{Sr}_{1.87} \mathrm{Ca}_{0.82} \mathrm{Cu}_{1.93} \mathrm{Bi}_{0.07} \mathrm{O}_{x}\right)(\mathrm{a})$ and $a=5.396 \AA, b=5.421 \AA, c=$ $30.51 \AA\left(\left(\mathrm{Bi}_{1.47} \mathrm{~Pb}_{0.52}\right)\left(\mathrm{Sr}_{1.79} \mathrm{Ca}_{0.04} \mathrm{Er}_{0.16}\right)\left(\mathrm{Ca}_{0.57} \mathrm{Er}_{0.43}\right)\left(\mathrm{Cu}_{1.94} \mathrm{Al}_{0.01} \mathrm{Bi}_{0.02}\right)_{x}\right)(\mathrm{b})$. The contributions to imaginary part of the dielectric function are shown by dashed-dotted curves.

samples composed of several isostructural 2212-type phases. Figure 1 shows the decomposition of the experimental Raman spectrum into the components which are characteristic of the co-crystallizing phases with $c$-values equal to 30.4 and $30.6 \AA$.

Figure 2 presents the dielectric function determined by spectroscopic ellipsometry method for two single crystals of different 2212-type isostructural phases. The first sample of orthorhombic symmetry exhibits an optically anisotropic feature at $3.65 \mathrm{eV}$ which could be taken as characteristics of the phase with the "large" c-parameter value. The optical response of the other sample with similar values of parameters $a$ and $b$ is less anisotropic and additional optical feature at $3.2 \mathrm{eV}$ can be revealed.

It should be noted that the main difference between the optical spectra of various isostructural phases occurs in the region of 3.5-4.5 eV which is caused by the electronic excitations in the $\mathrm{Bi}-\mathrm{O}$ plane. On the other hand, according to the structural data, the first sample showed the modulation of lattice parameter $b$. Additional studies have shown that just the features of the $\mathrm{Bi}-\mathrm{O}(\mathrm{Tl}-\mathrm{O}$ ) layers distinguish the structure of $\mathrm{Bi}-2212$ from that of related Tl-2212 compounds. 
The doping of the 2212-type phases by $\mathrm{Pb}$ and $\mathrm{RE}$ leads to the formation of several characteristic epitaxial intergrowths of isostructural phases: (i) thin films on single crystal substrate, (ii) alternating stacking of layers, (iii) complex single crystals with additional atomic layers. In the first case the complex sample can be represented as the single crystalline substrate of the stable 2212-type phase with the metastable 2212-type phase on the surface formed due to the presence of temperature gradient perpendicular to the surface.

The doped single crystals of type (ii) are wedge-shaped samples [3]. These "bulk" samples possess the properties characteristic of single crystalline formations, such as habitus, typical angles between the pseudo-faces, inclination to the twinning and well-defined $T_{\mathrm{c}}$ values. However, as followed from the structural and optical data, each sample face is the (ab)-plane of the crystalline 2212-type platelet. The EPMA data have shown that these samples represent the alternation of several isostructural phases of different chemical composition, when passing from the surface to the center of the sample. This type of intergrowth is most probably caused by a different local depletion of the basic components in the melt along the surface of the growing sample oriented perpendicularly to the $c$-axis.

Particular structural features of Bi-2212-type compounds are responsible for a formation of third version of epitaxial intergrowths in the single crystals with deviation from stoichiometry towards an increase in $\mathrm{Bi}$-amount. The investigation of these samples is of particular interest because the $T_{\mathrm{c}}$-values determined were up to $140-160 \mathrm{~K}$.

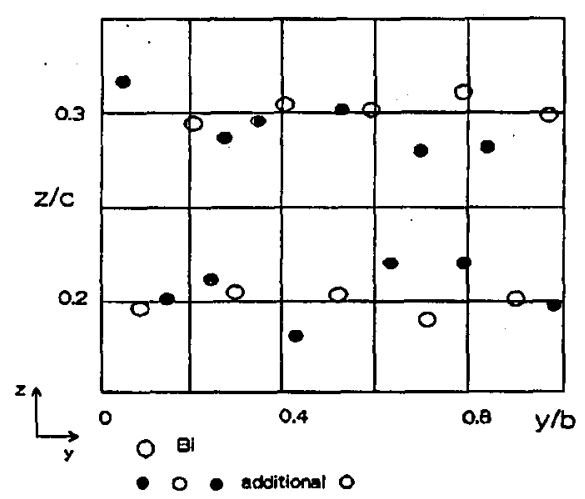

Fig. 3. The structure of $\mathrm{Bi}-\mathrm{O}$ layer of $\mathrm{Bi}_{2.00} \mathrm{Sr}_{1.56} \mathrm{Y}_{0.27} \mathrm{CaCu}_{2.00} \mathrm{O}_{8}(a=5.409 \AA$, $b=27.039 \AA, c=30.56 \AA$ ) single crystal analyzed in the $P n n n$ space group.

Analysis of the structure of the 2212-type single crystals (Fig. 3) allowed us to assume that the change in the chemical composition of the samples in group (iii) can be related to the deformation of the $\mathrm{Bi}-\mathrm{O}$ layer followed by a modulation of lattice parameters along the $y$-axis. As is seen in Fig. 3, the oxygen atoms in the $\mathrm{Bi}-\mathrm{O}$ layers tend to shift out of the plane according to the particular feature of $\mathrm{Bi}$-oxide structure. Besides, additional oxygens are inserted in the $\mathrm{Bi}-\mathrm{O}$ layer. As 
a result [5], the lattice parameter $b$ is modulated. The modulation is more clearly seen in the $\mathrm{Cu}-\mathrm{O}$ layer. Insertion of $\mathrm{Pb}$ leads to the reconstruction of rock-salt type structure of $\mathrm{Bi}-\mathrm{O}$ layer and to disappearance of structural modulation at the $\mathrm{Pb}$ concentration of $30 \%$ with respect to $\mathrm{Bi}$.

The distortion of $\mathrm{Bi}-\mathrm{O}$ layer towards the Bi-oxide structure allows one to assume that an excess of $\mathrm{Bi}$-content can lead to a formation of additional structural fragments. These fragments can be correlated with anomalously high $T_{\mathrm{c}}$-values in Bi-rich crystals of the 2212-type.

Formation of epitaxial intergrowths can be correlated with an increase in free carrier concentration. The optical investigations in the mid-infrared region allow one to scale the samples with respect to carrier concentration analyzing particular features of the dielectric function [6]. Basing on the optical data, a particular feature of some multiphase sample is to be noted: the carrier concentration is higher at an intergrowth of two isostructural phases as compared to that in the samples of each separate phases. It is reasonable to assume that a particular layered fragment is formed at the interface of the intergrowth of two phases with different lattice constants. The presence of this additional layer can be correlated with a higher carrier concentration of multi-phase samples.

Three types of epitaxial intergrowth considered are realized by different mechanisms caused by growth conditions, possibility to form the isostructural phases and specific structural features of $\mathrm{Bi}-\mathrm{O}$ layer. The intergrowths are typical of Bi-2212-type compounds due to their layered-type structure and are frequently met in the single crystalline samples.

\section{References}

[1] L.I. Leonyuk, A.G. Vetkin, E.L. Belokoneva, N.I. Leonyuk, Supercond. Sci. Technol. 5, 658 (1992).

[2] X.F. Zhang, G. Van Tendeloo, S.L. Ge, J.H.P.M. Emmen, V.A.M. Brabers, Physica $C 215,39$ (1993).

[3] L. Leonyuk, G.-J. Babonas, V. Maltscv, A. Vetkin, Supercond. Sci. Technol. 8, 53 (1995).

[1] L. Leonyuk, G.-J. Babonas, Vesınik MGU, Ser. 3 Fizika 1, 42 (1997).

[5] H.W. Zanderbergen, W.A. Grocn, F.C. Mijlhoff, G. van Tendeloo, S. Amelinckx, Physica C 156, 325 (1988).

[6] G.-J. Babonas, J. Bremer, R. Dagys, O. Hunderi, L. Leonyuk, G. Pukinskas, A. Vetkin, E. Wold, Supercond. Sci. Technol. 7, 479 (1994). 